\title{
Family Medicine Telehealth Clinic With Medical Students
}

\author{
Carolyn Peterseim | Kristen Hood Watson, MD
}

PRIMER. 2020;4:35.

Published: 12/21/2020 | DOI: 10.22454/PRiMER.2020.861306

\section{Abstract}

COVID-19 has altered clinical clerkships for medical students, providing an opportunity for telehealth with medical students to take center stage in the age of technology. This research brief demonstrates why and how to incorporate medical students into telehealth. For the purposes of clinical students, telehealth is a better educational alternative to in-person patient care than online modules. We performed a successful outpatient family medicine telehealth pilot program at the Medical University of South Carolina (MUSC) that is currently being scaled up to other clerkships. This pilot was very limited in scope, but serves as a scalable model for future telehealth programs and curricula incorporating medical students.

\section{Introduction}

Telehealth is an enhanced clinical learning opportunity for clinical medical students and a good substitute for in-person clinical time. ${ }^{1-4}$ Clinical time is defined by real-time interaction with real patients. Online modules do have certain utility for board review, but they are not an adequate clinical substitute. Through telehealth, students can see patients, write notes, learn from attendings, and train to operate with modern medical technology. Family medicine is ideal to facilitate telehealth clinical experiences for students, as many clinic visits are follow-up appointments with primary care providers, requiring small changes to their treatment plan.

There are some advantages to telehealth over in-person clinical time. Telehealth offers increased accessibility to patients and reduces transportation barriers to care. Providers and students can easily check a patient's chart during a clinic visit without disrupting it. Disadvantages include the inability to practice auscultation, percussion, and palpation on physical exams. It is possible to incorporate medical students into telehealth clinical visits. Those who struggle to adopt telehealth may consider other technologic advances they have adopted to care for patients. It will take effort, coordination, and teamwork, but now is the moment for us all to move forward and learn to operate with telemedicine.

\section{Methods}

Family medicine telehealth visits were held with students over the course of 3 months in the 2019-2020 school year. This initiative examined the efficacy of the program through average final National Board of Medical Examiners (NBME) Family Medicine Clerkship examination grades for blocks held in person and online. 
We used an online, free, secure app for FaceTime telehealth calls called Doxy.me to see patients with a family physician and medical student. There are many platform options, such as Vidyo and Cisco Webex. Attendings used Imprivata to remotely prescribe controlled substances. Telehealth calls integrated students through shadowing or presentation styles. Both methods used verbal consent of the patients before a medical student joined. Shadowing style added the student to the call with a short introduction. After the visit, the medical student and the attending were able to review the case in the virtual waiting room. Attendings could also ask the student to do a practice presentation, including their own ideas in their assessment and plan. Alternatively, a presentation style visit allowed the student to take the patient's history. Either the student called the patient before their appointment, or the student led the interview during the appointment. The medical student could also describe physical exam maneuvers to the patient to perform on themselves. For example, a patient may have dermatologic (eg, coloration, rash, bruising), respiratory (eg, coughing, wheezing, stridor, chest shape), cardiac (eg, thrill, jugular vein distention), gastrointestinal (eg, tenderness, distension, masses, jaundice), or focal neurologic findings observable on camera. Vitals may be obtained through patients' at-home monitoring devices, such as blood pressure cuffs.

We averaged final NBME grades for the eight in-person blocks prior to COVID-19 pandemic and compared to the two telehealth blocks, including the in-person blocks held in the previous school year at the same time of year (Table 1). We assessed these two groups using the nonparametric Mann Whitney $\mathrm{U}$ (Wilcoxon rank-sum test) for two independent samples, with the null hypothesis that the medians of these two groups are identical

$(P=.1)$. Each student in the two virtual blocks participated in at least two telehealth clinics (as described above) per week during their 6-week rotation. They were paired with a variety of family medicine attendings at Medical University of South Carolina (MUSC). Prior blocks included 5 days per week clinic with a variety of family medicine attendings in outpatient settings across South Carolina.

\section{Pilot Results}

Final NBME grades were equivalent in the telehealth and the in-person groups (median=80.2 +/- $95 \% \mathrm{Cl} 4.4$ vs $77.0+/-95 \% \mathrm{Cl} 2.1$ ) for 2019-2020 at MUSC's family medicine program. For our 2019-2020 comparison, we accept our null hypothesis that the difference between these two groups is not large enough to be statistically significant (Mann Whitney $U$ test $P=.533>0.1$ ). Limitations include that the telehealth groups occurred at the end of the school year and so it is possible that the average score was influenced by time of year, or that there were more students during the previous blocks of 2019-2020. However, we also compared the previous year's data from 2018-2019 for the final two blocks of the year, and found the telehealth group appear to have equivalent NBME average grades than the in-person blocks from the previous years (Table 2: $80.2+/-95 \% \mathrm{Cl}$ 4.4 vs $78.6+/-95 \% \mathrm{Cl} 0.4$ ). Another limitation is a small sample of telehealth blocks, and so this test is less likely to reject a null hypothesis.

\section{Conclusion}

Clinical knowledge can be obtained through telehealth, and students prepared through telehealth and in person performed equivalently on the NBME Family Medicine shelf exam. No online module can simulate the experience that comes with interacting with real patients, but this technology works to allow medical students to continue to see patients. Medical students learn to work through a patient's story in real time, practice documentation, and ask his/her attending questions about what medications are prescribed, as well as how to best support patients emotionally and physically during a pandemic. Telehealth is no simulation; it is real patient care. Future research can evaluate adding telehealth visits into COVID-19-modified clerkship curricula, as well as updating curriculum for medical students studying in the age of technology. 


\section{Tables and Figures}

Table 1: Comparison of National Board of Medical Examiners Grades

\begin{tabular}{|l|c|c|}
\hline & In Person & Telehealth \\
\hline Year & $\begin{array}{c}2019-2020 \\
\text { August-February } \\
\text { Blocks 1-6 }\end{array}$ & $\begin{array}{c}2019-2020 \\
\text { February-May } \\
\text { Blocks 7-8 }\end{array}$ \\
\hline Number of students & 138 & 45 \\
\hline Median grade & $77.0+/-95 \% \mathrm{Cl} 2.1$ & $80.2+/-95 \% \mathrm{Cl} 4.4$ \\
\hline & $P=.533>0.1$ & \\
\hline
\end{tabular}

Table 2: Comparison of National Board of Medical Examiners Grades (Final Two Blocks)

\begin{tabular}{|l|c|c|}
\hline Year & In Person & Telehealth \\
\hline Number of students & $\begin{array}{c}2018-2019 \\
\text { February-May } \\
\text { Blocks 7-8 }\end{array}$ & $\begin{array}{c}\text { 2019-2020 } \\
\text { February-May } \\
\text { Blocks 7-8 }\end{array}$ \\
\hline Median grade & 41 & 45 \\
\hline
\end{tabular}

\section{Corresponding Author}

\section{Carolyn Peterseim}

Medical University of South Carolina, College of Medicine, Family Medicine, 5 Charleston Center Drive, Suite 263, Charleston, SC 29425

\section{Author Affiliations}

Carolyn Peterseim - Medical University of South Carolina, College of Medicine, Family Medicine, Charleston, SC Kristen Hood Watson, MD - Medical University of South Carolina, College of Medicine, Department of Family Medicine, Charleston, SC

\section{References}

1. Waseh S, Dicker AP. Telemedicine training in undergraduate medical education: mixed-methods review. JMIR Med Educ. 2019;5(1):e12515. doi:10.2196/12515

2. Theobald M, Brazelton T. STFM forms task force to develop a national telemedicine curriculum, from STFM. Ann Fam Med. 2020;18(3):285-286. doi:10.1370/afm.2549

3. Aungst TD, Patel R. Integrating digital health into the curriculum-considerations on the current landscape and future developments. J Med Educ Curric Dev. 2020;7:2382120519901275.

doi:10.1177/2382120519901275

4. Rhone K, Lindgren J, Mack LJ, Nelson ME, Spencer L, Anderson SM. Avera eCARE: medical student education in telemedicine. Telehealth and Medicine Today. 2020. 5(2) Published April 29, 2020. doi:10.30953/tmt.v5.179 